\title{
Analysis of recent empirical challenges to an account of interceptive timing
}

\author{
JAMES R. TRESILIAN \\ University of Queensland, Brisbane, Australia
}

\begin{abstract}
How do we perceive how long it will be before we reach a certain place when running, driving, or skiing? How do we perceive how long it will be before a moving object reaches us or will arrive at a place where it can be hit or caught? These are questions of how we temporally coordinate our actions with a dynamic environment so as to control collision events. Much of the theoretical work on the control of these interceptive actions has been united in supposing that (1) timing is functionally separable from positioning and the two are controlled using different types of information; (2) timing is controlled using special-purpose time-to-arrival information; (3) the time-to-arrival information used for the timing of fast interceptive actions is a first-order approximation to the actual time-to-arrival, which does not take accelerations into account. Challenges to each of these suppositions have recently emerged, suggesting that a complete rethinking of how interceptions are controlled may be necessary. These challenges are analyzed in detail and it is shown that they are readily accommodated by a recent theory of interceptive timing based on the points just noted.
\end{abstract}

Interceptive actions like catching or hitting a ball have both positional and timing requirements: To catch a ball, the hand must be in the right place at the right time. Since a person can make timed responses in the absence of any positional requirements and positional responses in the absence of any external timing requirements, it is possible to make a functional distinction between timing (when) and positioning (where). Probably because of this, it has been supposed that timing and positioning can be considered separable components of interception, controlled by different sources of information: Timing information tells you when something needs to be done and positional information tells you where to do it.

A major theoretical contribution to understanding the timing component was Lee's original "tau hypothesis," described in his earlier publications (Lee, 1980; Lee \& Young, 1985; Lee, Young, Reddish, Lough, \& Clayton, 1983). Although the tau hypothesis has nowhere been explicitly stated in full by Lee, it is generally supposed to consist of the following propositions:

1. Interceptive actions are timed using information about time-to-arrival or time-to-collision (TTC); this information is provided by the variable tau $(\tau)$, which has been defined most frequently as the ratio of image size to rate of change of size (Lee, 1976): the reciprocal of the relative rate of image expansion.

2. Proposition 1 applies to all interceptive actions and has the important corollary that accelerations are ignored in estimating TTC (Bootsma \& Oudejans, 1993; Lee et al., 1983).

Correspondence should be addressed to J. R. Tresilian, Department of Human Movement Studies, University of Queensland, St. Lucia, Brisbane, QLD 4072, Australia (e-mail: jamest@hms.uq.edu.au).
3. Particular fixed values of tau, often referred to as margin values (Lee, 1980; Lee \& Reddish, 1981), are used to initiate movements and subsequent movement events (Lee \& Reddish, 1981; Van der Weel, Van der Meer, \& Lee, 1996). In addition, the timing of many actions is continuously regulated during execution by tau (Lee et al., 1983).

These three propositions have a deceptive simplicity that lends the tau hypothesis a certain attraction: All that timing of any interception requires is that a simple optical quantity be measured and movements "geared" to the value of this quantity (Lee \& Young, 1985). Unfortunately, a careful analysis of empirical data and the temporal precision requirements of interceptive actions has revealed that none of these propositions can be generally correct. Proposition 1 is refuted by the observation that tau is incapable of providing the TTC information necessary for intercepting moving targets that pass a person some distance from the eyes (Tresilian, 1990, 1994a). Proposition 2 is refuted by the observation that certain interceptions of objects falling short distances under gravity are timed using relatively accurate estimates of TTC, and so accelerations must be being taken into account (Lacquaniti \& Maioli, 1989; see also Tresilian, 1995a, 1997b, and Wann, 1996).

The first part of Proposition 3 is refuted by the observation that interceptive actions are seldom initiated at fixed values of tau or TTC. Instead, it has been repeatedly observed that movements are initiated earlier and take longer to execute when the target moves more slowly and are initiated later and are executed more quickly when the target moves faster (Bootsma \& van Wieringen, 1990; Brenner \& Smeets, 1994; Laurent, Montagne, \& Savelsbergh, 1995; Wallace, Stephenson, Weeks, \& Kelso, 1992). Indeed, it has not proved to be generally possible to identify a variable that has a fixed value at the time of move- 
ment initiation in interceptive timing. A plausible explanation for this observation is that the nervous system does not initiate movements at a fixed value of any variable after all, why should it?-instead, a more flexible strategy is employed in which a value of TTC is used to initiate movement, but this value depends on the circumstances of execution and "internal" factors. The value not only initiates the action but is also used to set the movement execution time since the latter must be appropriate for the TTC at initiation; otherwise, accurate interception would not be possible. This strategy is more flexible because a person does not need to initiate movement at a fixed TTC; movement can be initiated earlier or later, depending on whether this would facilitate performance. In particular, it overcomes the following problem with the fixed value strategy: Unless the value is the smallest possible (i.e., the person initiates the fastest movement possible at the last possible moment), the performer necessarily gives up the ability to make interceptions that could be made and has no control over the speed of execution. If the smallest value is used, the performer must always execute the movement as fast as possible and with great accuracy since there is no longer any room for error. This has an important implication for data analysis. A method that has been widely used to analyze data from interceptive actions in order to determine which of several candidate control variables is responsible for the initiation of a movement event is to compare their unitless coefficients of variability (CVs). The $\mathrm{CV}$ is defined as the standard deviation divided by the mean, and the variable with the smallest $\mathrm{CV}$ at the time of movement event initiation is identified as the variable controlling initiation (Davis \& Green, 1990; Van der Weel et al., 1996). This method assumes that movement events are initiated at fixed values of control variables - if a control variable has a fixed value at initiation, then its variability at the moment of initiation should be very small. Since there is no reason to suppose that fixed values of control variables are used to initiate movement events, and good reasons to suppose otherwise, this method of analysis is clearly inappropriate. It is not possible to determine the control variable by comparing the CVs of the various possibilities and choosing that with the smallest $-\mathrm{CVs}$ cannot be used to determine control variables.

\section{A New Hypothesis for Interceptive Timing}

One possible response to the problems with the tau hypothesis is to develop a new hypothesis for interceptive timing that specifically deals with the problems. Lee has attempted to deal with some of these problems. For example, in Lee and Young (1985), he tackled the problem that tau as originally defined provides information about TTC with the eye; his approach inspired my own general solution to the problem (Tresilian, 1990). However, his approach appears to be to search for a general solution to the problem of interceptive timing, and as such has been largely unsuccessful. The analyses that I have conducted (Tresilian, 1991, 1994a, 1995a, 1997b) strongly suggest that a general solution is not possible-certainly not one based on tau functions. For this reason, my approach is to reject both the idea that all interceptive actions are timed using the same information and the idea that other tasks that may involve TTC estimates necessarily involve similar information to that used to time interceptions. These other tasks include relative judgment tasks (which of two moving objects will arrive at a designated location first?) and prediction motion tasks (the terminal phase of a moving object's trajectory is occluded and the observer must indicate the moment when the unseen object would reach a designated location).

Instead of proposing a single timing strategy for all interceptive actions and TTC judgments, I have suggested that the strategy used is task dependent and that judgment tasks should be carefully distinguished from interceptive actions, since these two classes of task may involve quite different streams of visual processing (Tresilian, 1995a). Even within the class of timed actions (the class of tasks considered in this article), timing in different tasks may involve different strategies (different sources of information and different ways of using this information to control responding). It is not suggested, however, that the different strategies are arbitrary or unrelated. Rather, they are related by being the result of the operation of systematic constraints on a system that learns and adapts itself to different circumstances. Such a system will learn to use whatever information is available in any way it can to achieve satisfactory performance. Under this conception, the simple elegance of the tau hypothesis is lost. Nevertheless, it is consistent with the data on interceptive timing (Tresilian, 1994b, 1995a) and with what we know about nervous systems-these care nothing for elegance or optimality; rather, they are concerned with finding satisfactory solutions to behavioral problems (see, e.g., Ramachandran, 1990). Solutions will approach elegance and optimality only to the extent that satisfaction of performance demands requires it.

This conception of the human performer of an interceptive action suggests that it is important to understand what an interceptive task demands of a person and what information is available to satisfy these demands. As a step in this direction, it is possible to identify an important group of interceptions that are characterized by short movement times (MTs; $\leqslant 0.5 \mathrm{sec}$ ), brief target-viewing periods $(\leqslant 1 \mathrm{sec})$, and requirements for very precise timing (better than $\approx 30 \mathrm{msec}$ ). Such actions occur frequently in ball sports and may be referred to as fast interceptive actions. An example would be playing a fast ball in baseball or cricket. In the latter, MT is on the order of $300 \mathrm{msec}$ (McLeod, 1987); after it leaves the bowler's hand, the ball is seen for less than about $700 \mathrm{msec}$ before being struck, and the temporal precision required for a good stroke has been estimated to be on the order of $\pm 2.5 \mathrm{msec}$ (Regan, 1992).

The performance demands of many fast interceptive actions, coupled with the known insensitivity of the human 
visual system to image accelerations, suggests that people use TTC information to achieve precise timing in these tasks and that the information used is a first-order estimate of the actual TTC that does not take target acceleration into account (Tresilian, 1993, 1994a). ${ }^{1}$ (Note that this hypothesis does not apply to interceptions of objects falling a short distance under gravitational acceleration: In such circumstances, first-order TTC estimates are useless for accurate timing (see Tresilian, 1997b, for further discussion). Tau is seldom sufficient to provide the TTC information necessary for precise timing in these tasks, but it has been shown that there are a variety of sufficiently precise sources of information about first-order TTC (Tresilian, 1990, 1993; see below). Thus, for fast interceptive actions, Propositions 1-3 of the tau hypothesis are replaced by the following:

$1^{\prime}$. Many fast interceptive actions are timed using a firstorder estimate of TTC. There are a variety of sources of this information, and those used will depend on a variety of factors, including timing constraints, type of task, and the performer's familiarity with the task.

$2^{\prime}$. Any accelerations acting do not affect speed significantly during the short time periods available for the actions to which $1^{\prime}$ applies. Thus, a first-order estimate of TTC is sufficient. Other actions may involve the use of a variety of different cues (e.g., distance cues, cues to target speed) and possibly acceleration information (Tresilian, 1995a; see below).

$3^{\prime}$. Movement events are not initiated at fixed values of TTC or some other informational variable. Movements are initiated flexibly in a fashion that depends on the circumstances of execution, which are likely to include (1) the effector being used (arm, foot, arm/bat, etc.--an effector with greater inertia may require more time to get it moving and cannot generally be moved as fast as one with smaller inertia. This will tend to make it necessary to initiate movement commands rather earlier to more massive effector systems. More distal effector systems will also involve greater neural transmission time delays; although these will only be of the order of a few milliseconds many interceptive actions require millisecond precision; see, e.g., Regan, 1992); (2) the distance to be moved; (3) the timing constraints; and (4) characteristics of the target (speed, mass, size).

Thus, for fast interceptive actions, this account accepts two basic premises of the tau hypothesis: (1) Timing is a separable component of interceptive action controlled by TTC information, and (2) many actions are timed using a first-order estimate of TTC that ignores accelerations. This hypothesis is supported by very little direct empirical data and demands rigorous empirical testing. Recently, several experimental reports have been published that appear to be inconsistent with various aspects of the hypothesis just described. First, DeLucia (DeLucia \& Meyer, 1997, personal communication, November 1996) observed that the first-order TTC information described in the literature (Bootsma \& Oudejans, 1993; Tresilian, 1990, 1993) has been derived under the assumption of a stationary observer, and she has pointed out that certain observer motions may compromise the utility of this information. Recent results have demonstrated that people are able to estimate TTC during simulated observer motions (DeLucia \& Meyer, in press). Second, Smeets and colleagues have challenged the proposal that timing involves the use of TTC information per se, proposing instead that TTC is computed from independently derived information about distances and velocities in external 3-D space (Smeets, Brenner, Trebuchet, \& Mestre, 1996). Third, the notion that image accelerations are ignored in any fast interceptions has been questioned by Stewart, Cudworth, and Lishman (1993, in press). Fourth, a theory of projectile interception that has recently become popular supposes that positioning and timing are not separable components of interception. This is a servo control theory based on perception of the acceleration of the tangent of the projectile's elevation angle (Chapman, 1968; McLeod \& Dienes, 1993, 1996; Micheals \& Oudejans, 1992; Tresilian, 1995b; see also McBeath, Shaffer, \& Kaiser, 1995, for a similar and related proposal).

In what follows, each of these three issues and empirical data that relate to them will be discussed in detail to determine whether a first-order, TTC-based understanding of fast interceptive timing should be rejected.

\section{INFORMATION}

It has been established that there are sources of firstorder TTC information that can provide a stationary observer with information about when a moving object will reach an arbitrary fixed location on its trajectory (Bootsma \& Oudejans, 1993; Tresilian, 1990, 1991). This information involves the rate of change of the angle between the visual directions of the object and the upcoming collision location. Recently, questions have been raised concerning how perception of TTC is affected by observer motion, which will cause the visual angle subtended by the gap between object and collision location to change independently of object motion (DeLucia \& Meyer, 1997). The information described in Tresilian (1990) and Bootsma and Oudejans (1993) assumes a stationary observer, but motion of the observer may compromise the accuracy of these sources of information. This is clearly an important issue since the point of observation $(O)$ is rarely, if ever, stationary-people often catch balls on the run (see below), and DeLucia and Meyer (in press) have presented data that often showed a similar accuracy for TTC estimates for stationary and moving observers in a prediction motion task.

Here we extend this work to examine the more general case of TTC perception for a moving observer. Consider the geometry shown in Figure 1. From this geometry we can write down the following: $\sin \beta(t) / R_{p}(t)=\sin \psi(t) / X(t)$. Taking the temporal derivative (and dropping functional dependence on time, $t$, from the notation) gives us $\dot{X} \sin \beta$ $+\mathrm{X} \dot{\beta} \cos \beta=\dot{R}_{p} \sin \psi+R_{p} \dot{\psi} \cos \psi$. Dividing these two re- 


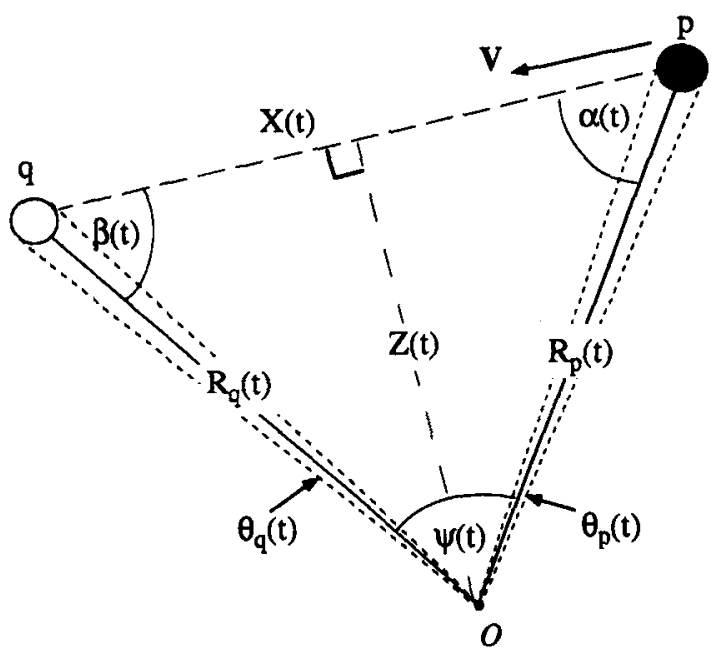

Figure 1. Planar monocular optic geometry of collision between two "objects," $p$ and $q$, moving along straight line paths.

lations and using the notation $-R / \dot{R}_{p}=\theta_{p} / \dot{\theta}_{p}=\tau\left(\theta_{p}\right)$ and $-X / \dot{X}=$ TTC allows us to derive the following:

$$
\mathrm{TTC}=\tau\left(\theta_{p}\right) /\left[1-\tau\left(\theta_{p}\right)(\dot{\psi} \cot \psi-\dot{\beta} \cot \beta)\right] .
$$

Here TTC is the time-to-collision of the two objects, $p$ and $q$, if they are on a collision course. If they are not going to collide, then TTC is the time at which the visual directions of $p$ and $q$ will coalesce-if one object, say, $p$, passes in front of the other ( $q$ ), TTC is the time-to-occlusion of $q$ by $p$. Equation 1 is true for the case when $p, q$, and $O$ are moving. Equation 1 has a form that is difficult to interpret. It is easier to present it in terms of the immediacy of contact-the reciprocal of the time-to-contact (see Koenderink, 1985): If the immediacy of collision between $p$ and $q$ is $\Gamma_{p q}$, then Equation 1 can be written as follows:

$$
\Gamma_{p q}=\tau^{-1}\left(\theta_{p}\right)-\dot{\psi} \cot \psi+\dot{\beta} \cot \beta
$$

The immediacy of contact will often be used in preference to TTC because of the notational advantages it confers. In Equations 1 and $2, \tau\left(\theta_{p}\right)$ is the relative rate of dilation of the visual angle subtended by $p ; \psi$ and $\dot{\psi}$ are, respectively, the angle subtended by the "gap" between $p$ and $q$ and its rate of change. All these variables can be readily measured by the visual system because they are directly available at $O$. This is not the case for $\beta$-the angle between the visual direction of $p$ and the direction of relative motion of $p$ and $q$-or the rate of change of $\beta(\dot{\beta})$ : These quantities cannot be measured directly by a monocular observer. Equations 1 and 2 can be reduced to simpler forms that involve only quantities available monocularly if either $\beta$ is constant or $O$ is stationary.

\section{Case 1, $\beta$ Constant}

Under the following conditions, $\beta$ is constant: (1) Both $O$ and $q$ are stationary. (2) $O$ is moving directly along the line
$O q$, which could arise because $q$ is stationary and $O$ is moving along $O q$, or $q$ could be moving with $O$ always moving in the direction of $q$. Under these conditions, the third term on the right-hand side of Equation 2 is zero, and it reduces to an equation first presented in Tresilian (1990) to demonstrate how TTC with an arbitrary fixed visual direction is specified to a stationary observer: $\Gamma_{p q}=\tau^{-1}\left(\theta_{p}\right)-\dot{\psi} \cot \psi$. If we add the additional assumption that $\psi$ is small, this reduces to $\Gamma_{p q} \approx \tau^{-1}\left(\theta_{p}\right)-\dot{\psi} / \psi$ (Bootsma \& Oudejans, 1993), though this assumption is seldom justified.

DeLucia and Meyer (1997) have presented data for the case of an observer moving relative to a moving object on a collision course with a stationary object. In the experiments, a camera moved in such a way that $\psi$ was zero (the "optical gap" between the target objects is unchanging). Experimental participants were often able to judge TTC in this condition with similar accuracy to that observed in the absence of observer motion. This case is the simplest of all since both $\beta$ and $\psi$ are constant and Equation 2 reduces to $\Gamma_{p q}=\tau^{-1}\left(\theta_{p}\right)$. This case is optically identical to that in which two objects approach a stationary observer from different directions in such a way that they would hit him/her at the same moment.

\section{Case 2, $O$ Stationary}

Here $\beta$ is not constant when both objects, $p$ and $q$, are moving. Note, however, that $Z(t)$ is now constant: We have $\sin \beta=Z / R_{q}$; taking derivatives and using the same notation as before; we can establish that $\tau^{-1}\left(\theta_{p}\right)=\dot{\beta} \cot \beta$. This we can substitute for $\dot{\beta} \cot \beta$ in Equation 2 to get

$$
\Gamma_{p q}=\tau^{-1}\left(\theta_{p}\right)+\tau^{-1}\left(\theta_{q}\right)-\dot{\psi} \cot \psi
$$

\section{Case 3, Binocular Observer}

It is straightforward (though rather laborious) to demonstrate that a binocular observer can obtain the information in Equation 1 entirely from visually measurable variables for the case of two objects on a collision course. For the sake of completeness, an outline of the argument that establishes this will be provided with reference to Figure 2 .

Referring to the diagram in Figure 2 and using the sine rule, we can write down the following:

$$
D_{L}=I \sin \phi_{R} / \sin \gamma_{q} \text { and } R_{L}=I \sin \left(\phi_{R}+\psi_{R}\right) / \sin \gamma_{p} \text {, }
$$

where $I=$ interpupilliary distance. The cosine rule states that $X^{2}=D_{L}^{2}+R_{L}^{2}-2 D_{L} R_{L} \cos \psi_{L} ;$ substituting for $D_{L}$ and $R_{L}$ yields

$$
\begin{aligned}
(X / I)^{2}= & \left(\sin \phi_{R} / \sin \gamma_{q}\right)^{2}+\left(\sin \left[\phi_{R}+\psi_{R}\right] / \sin \gamma_{p}\right)^{2} \\
& +2 \sin \phi_{R} \sin \left(\phi_{R}+\psi_{R}\right) \cos \psi_{L} /\left(\sin \gamma_{q} \sin \gamma_{p}\right)
\end{aligned}
$$

Taking the temporal derivative of Equation 4 gives $2 X \dot{X} /$ $I^{2}$ for the left-hand side. Dividing the temporal derivative of Equation 4 by Equation 4 gives, on the left-hand side, $2 X / X$-twice the immediacy of contact $\left(2 \Gamma_{p q}\right)$. The right- 


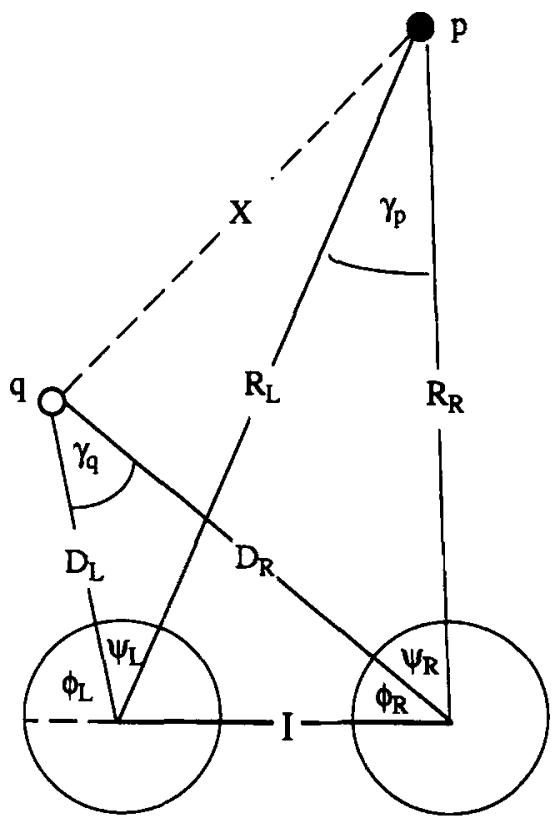

Figure 2. Planar binocular optic geometry of collision: $p$ and $q$ are (moving) objects on a collision course and have instantaneous binocular parallax $\gamma_{p}$ and $\gamma_{q}$, respectively. The instantaneous direction of the relative motion of $p$ and $q$ with respect to the line joining the centers of the two eyes is $\alpha$. The interocular separation is $I$.

hand side is a complex expression involving the variables $\phi_{R}, \psi_{R}, \gamma_{q}, \gamma_{p}$, and their temporal derivatives. This argument establishes that a binocular observer can obtain information about the TTC of two objects from retinally measurable variables. However, it may be relatively unusual for two objects to be on a direct collision path, and when they are, it is not necessarily useful to have accurate estimates of the TTC. The argument also applies, however, to the possibly more useful case of one object moving toward a stationary collision location for arbitrary constant velocity motion of the observer.

These cases suggest that an observer will have problems obtaining an accurate estimate of the time-to-occlusion of two objects moving with constant velocity that will not collide (see, e.g., DeLucia, 1995). A binocular or a stationary monocular observer can, in principle, determine the first-order TTC of two such objects when they are on a collision course, but a moving monocular observer will have problems. Provided that the direction of an interception location remains constant relative to a moving observer, accurate first-order TTC information can be obtained for a moving object. Thus, the general question reveals that there may be informational limitations on a human observer's ability to obtain accurate estimates of first-order TTC under certain conditions.

\section{WHAT INFORMATION DO PEOPLE USE?}

In this section, three questions will be considered: (1) Do people use TTC information per se such as that described above, or do they compute TTC from independently obtained distance and velocity information? (2) Do people use both monocular and binocular sources of TTC information? and (3) How general is the strategy of ignoring accelerations in interceptive timing?

\section{Do People Use a Distance Divided by Velocity Strategy?}

In a recent article Smeets et al. (1996) set out to provide us with an answer to the following question: Is TTC of a moving target with a (stationary) contact point derived from optical variables or is it "computed" by dividing an estimate of the distance between the target and contact point by an estimate of the target's speed? The data presented by Smeets et al. were interpreted as establishing that judgment of TTC "is primarily based on the ratio between the perceived distance and the perceived velocity" (p. 583).

Smeets et al. (1996) used a computer-generated display of a target object moving along a straight-line path in the plane of the screen toward a contact line. The target moved over a sparse background of randomly positioned short line segments that were either stationary or moved together as a group. Changes in the relative motion of target and background influence the apparent speed of the target: It appears to move faster when the background moves in the opposite direction and slower when the background moves in the same direction. Smeets et al. reasoned that if TTC judgments were influenced by background motion in a manner consistent with the effect on apparent speed of the target, this would establish that observers were measuring TTC from a combination of speed and distance information. The results suggested that apparent speed was indeed influencing TTC in the expected fashion. Smeets et al. interpreted this to mean that observers were estimating TTC on the basis of the target's perceived speed in 3-D space and, by implication, the perceived distance.

The most crucial aspect of this interpretation of the results is the assumption that stimulus information is used (along with some scaling factor) to generate a perceptual representation of the target's actual speed through external space, and it is this representation that is used to compute TTC. It is not necessary, however, to make this assumption: Suppose that a person were using the TTC information in Equation 3 to make judgments in Smeets et al.'s (1996) paradigm. To use this information, it is necessary to measure $\dot{\psi}$ : How does the visual system do this? When the background is moving, the visual system must somehow segregate sensed motion into motion due to the target's movement $(\dot{\psi})$ and motion due to movement of the background. If, for whatever reason, the visual system does not do this perfectly, then background motion is expected to influence measurement of $y$ and hence of TTC. Presumably, perceived target speed in space depends on $\dot{\psi}$. Thus, both speed and TTC could be influenced by background motion in the same fashion because of their dependence on the measurement of $y$ and not, as Smeets et al. have argued, because TTC perception depends on the prior perception of target speed. 
Use of Equation 2 and related expressions as information about TTC requires that the optical speed of the target be measured perceptually - an argument I have made previously and supported with experimental data (Tresilian, 1994a). Smeets et al.'s (1996) results can be interpreted as demonstrating that measurement of optical speed is influenced by background motion, and this is quite consistent with the account of TTC perception I have presented (e.g., Tresilian, 1994a). Thus, Smeets et al.'s claim that their results cannot be explained by existing models of TTC perception, which are based on optical quantities, cannot be substantiated: Their results do not address the question of whether optical speeds are used in the computation of TTC; rather, they can be viewed as addressing the question of how optical speeds are measured by the visual system.

The distance-divided-by-velocity account of TTC has a number of problems. First, there are no data to support it over the alternative under discussion. Second, we know that in the absence of absolute distance and velocity information, human observers can make accurate TTC judgments, establishing that purely optical sources of information can be used (e.g., Kaiser \& Mowafy, 1993; Regan \& Hamstra, 1993; Schiff \& Detwiler, 1979; Todd, 1981). To accommodate these data, the distance-divided-byvelocity account must assume that the human visual system is able to switch to use of purely optical information when absolute information is unavailable. When absolute information is available, it is used in preference to purely optical information, but no reasons for preferring it have ever been put forward. Indeed, there are at least two good reasons for rejecting the idea: First, it introduces additional sources of error in the form of the scale factors used to derive the absolute information from the purely relative information directly available to the senses: Any error in the values of these scale factors will lead to additional error in TTC estimation. Second, scaling into absolute terms is a completely unnecessary operation that is effectively undone when distance and velocity are divided -its effect can only be to increase the error in TTC estimation. Why should the nervous system bother to perform an operation only to undo it again, achieving only increased error in the process? Clearly, the distance-divided-by-velocity account is in need of considerable development before it can be meaningfully compared with the alternative discussed here, especially since no one has ever put forward an empirical means for distinguishing it from a purely sensory account.

An important final point should be made concerning the methodology used by Smeets et al. (1996) in their experiment: A TTC judgment task was employed, not an interceptive action. It is well established that many types of TTC judgment and estimation tasks elicit responses that show quite different temporal error characteristics from those observed in actual interceptions (see Tresilian, 1995a, for discussion). Available data strongly support the hypothesis that the information used in making various types of TTC judgment is strongly task dependent and may be quite different from that used to control interceptions. This being so, it is not legitimate to test the hypothesis for interceptive timing described in this article using tasks that are not themselves interceptive actions. Thus, although Smeets et al,'s results are quite consistent with the use of the types of TTC information described elsewhere (e.g., Bootsma \& Oudejans, 1993; Tresilian, 1990, 1991), they may not be replicated when an interceptive action is used in place of a judgment task.

\section{Constant Velocity?}

Since velocity is a vector, there are two possible parts to the constant velocity hypothesis: The part proposed by Lee (1976) is that a changing magnitude of the velocity vector (speed) is not taken into account in computing TTC. The second part would be the proposition that a changing direction of the velocity vector is not taken into account either. The present discussion will be restricted to the first part, leaving discussion of the second for the future.

The hypothesis that people ignore accelerations in the computation of the TTC information used to time fast interceptive actions applies to all such actions with the exception of intercepting objects falling a short distance under gravity (Tresilian, 1995a, 1997b). The hypothesis has been underresearched and awaits definitive empirical tests. What can be said is that many fast interceptive actions can be adequately timed even if accelerations are ignored. This in no way establishes that accelerations are ignored, but there are three arguments that make the hypothesis a plausible proposition:

1. Most interceptions that do not involve approximately vertical falls under gravitational acceleration rarely involve predictable, appreciable changes of velocity within the time frame of an interception. This is true even of vertical falls under gravity of more than a few meters (see Lee $\&$ Young, 1985). Figure 3 shows the approximation provided by first-order TTC information plotted as a function of the actual TTC for variations in the falling distance. Data suggest that corrections to ongoing actions can be made up until about $100 \mathrm{msec}$ prior to movement completion (collision). The diagram reveals that timing to within 10 msec could be achieved in such actions for drop heights in excess of about $3 \mathrm{~m}$. Actions that are executed rapidly (MTs of less than about $150 \mathrm{msec}$ ), such as a swipe with the hand or a bat like the swing of a smash shot in table tennis, or perhaps the grasp phase of a catch, would be initiated perhaps $250 \mathrm{msec}$ or less before contact and may be ballistic.

Figure 3 suggests that ballistic timing of interceptions of falling objects to within about $25 \mathrm{msec}$ accuracy would be possible for falls greater than about $5 \mathrm{~m}$. Accurate timing (within $50 \mathrm{msec}$ ) for falls less than $2-3 \mathrm{~m}$ is not possible using first-order TTC. Where changes in velocity do occur in situations other than falls under gravity, they are almost always due to external disturbances and so cannot be predicted from the previous behavior of the moving target. For example, in the absence of external perturbations, 

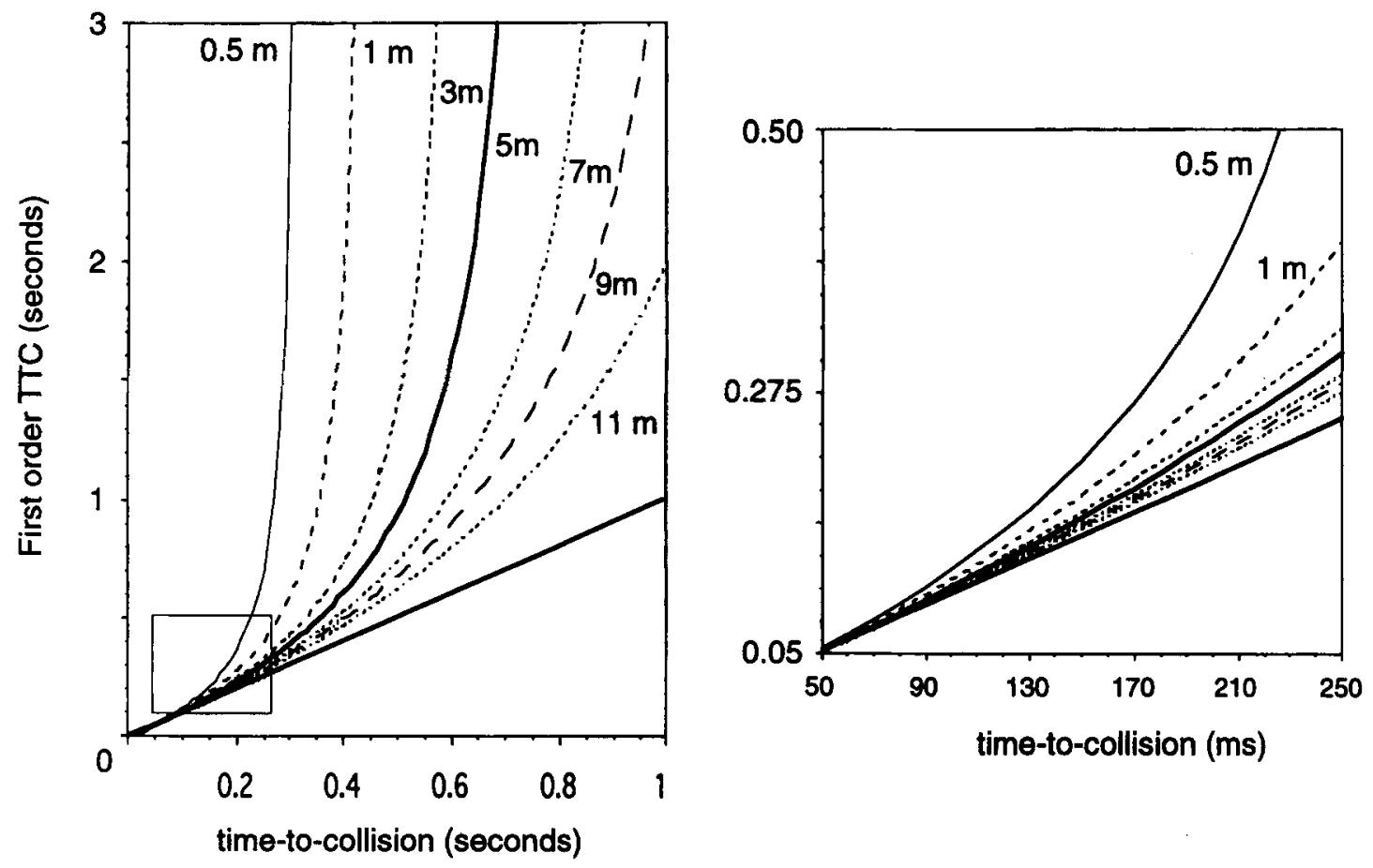

Figure 3. First-order time-to-collision (distance from collision point divided by speed) as a function of actual time-tocollision for objects falling from different heights above the collision point $(11,9,7,5,3,1$, and $0.5 \mathrm{~m})$ under gravity with negligible air resistance. The solid straight line is the first-order TTC $=$ actual TTC line. The right panel shows an expanded view of the rectangular insert in the left panel.

a baseball pitch or a tennis stroke does not change velocity along the direction of bat racket contact to a degree sufficient to significantly influence TTC in the time available.

2. Extracting TTC when the target is accelerating requires that image accelerations be measured by the visual system. It has been found that the human visual system is very insensitive to image accelerations, requiring a change in speed of at least $20 \%$ (see, e.g., Babler \& Dannemillar, 1993; Regan et al., 1986; Werkhoven et al., 1992). This insensitivity is such that over the short viewing times typical of fast interceptions, image acceleration information could not serve to improve timing precision. Note that when there is acceleration due to gravity, image accelerations do not need to be measured to obtain an accurate estimate of TTC - the acceleration due to gravity is a terrestrial constant, the value of which could be learned and internalized through experience.

3. The available data from fast interceptive actions are consistent with the hypothesis that accelerations are ignored in fast interceptions that do not involve relatively short falls under gravity (Tresilian, 1993, 1995b)-for short falls, gravitational acceleration appears to used (Lacquaniti \& Maioli, 1989; Tresilian, 1997b).

Argument 1 is based on the ecology of interception: The largest predictable acceleration in the natural world is that due to gravity, which acts in the vertical direction. Other types of interceptions that would occur when an organism is running, walking, or preying on other animals involve accelerations that are much smaller in magnitude than that due to gravity, and most of these are unpredictable from prior motion. Argument 2 is based on psychophysical results concerning human insensitivity to image accelerations. If image acceleration is not suprathreshold, then it cannot contribute to TTC perception, and it is unlikely to reach threshold under the conditions characteristic of most fast interceptive actions. It is possible that this insensitivity is a direct consequence of Argument 1: It is not necessary to equip a visual system with the ability to quickly and accurately measure image accelerations, since such an ability would not confer a significant survival (reproductive) advantage.

Recently, Stewart et al. $(1993,1997)$ challenged the idea that image accelerations do not contribute to the information used in timing fast interceptions. They proposed instead that timing is based on the tau-like quantity $\dot{\theta} / \vec{\theta}$ - the tau function of $\dot{\theta}, \tau(\dot{\theta})$-defined in Figure 4. In the geometry shown in Figure 4, this quantity has an advantage over tau-it provides a much better approximation to the actual TTC than tau when the target is accelerating. I have analyzed Stewart et al.'s claim that $\tau(\dot{\theta})$ is the source of information used in interceptive timing (Tresilian, 1997b). I generalized $\tau(\dot{\theta})$ to cases other than that originally considered by Stewart et al. (top of Figure 4 ) in order to determine whether $\tau(\dot{\theta})$ could provide a viable alternative to tau. I was forced to conclude that $\tau(\dot{\theta})$ is no better than tau since it provides TTC information with an observer's 

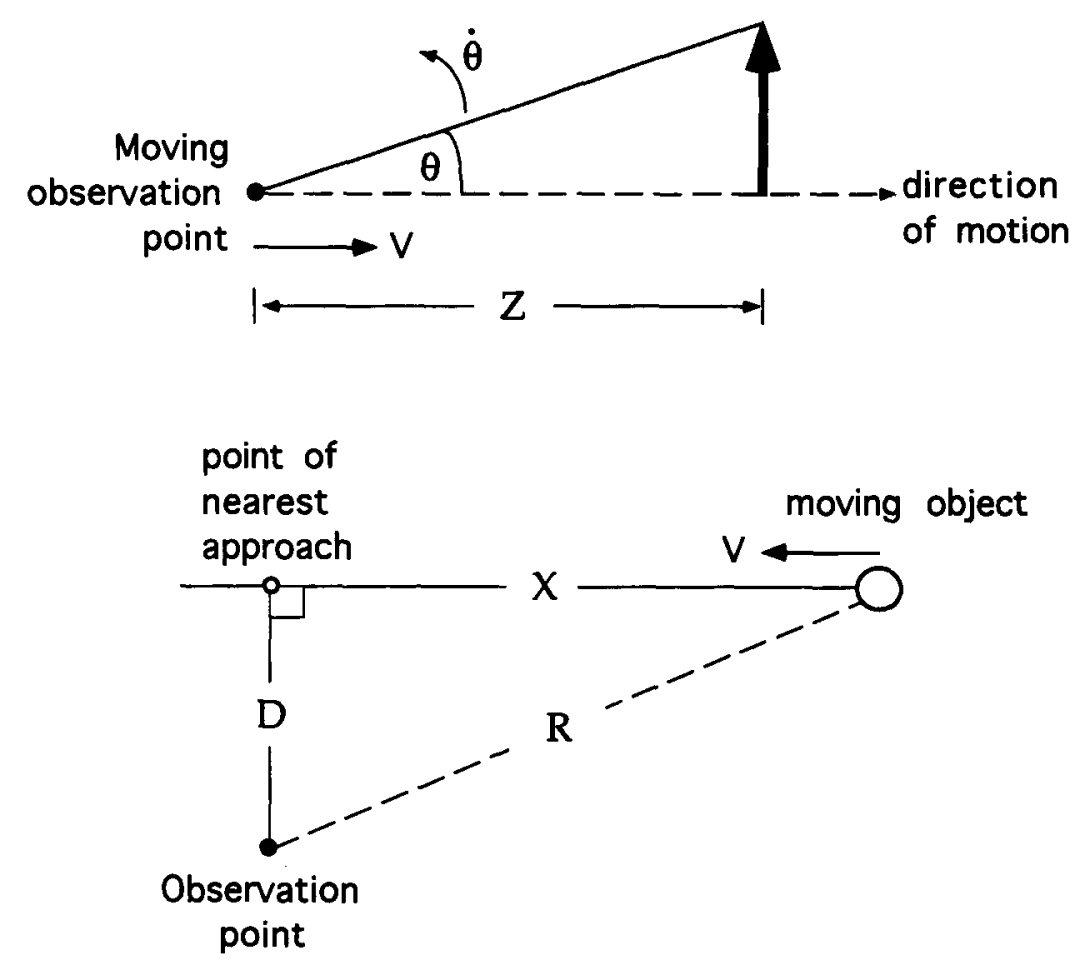

Figure 4. Optic geometry of time-to-collision of a moving observer with a stationary object (top) and time to nearest approach of a moving object with a stationary observer (bottom). The case shown at the top is that treated by Stewart et al. (1993), who showed that $\dot{\theta} / \ddot{\theta}=\tau_{A}(\dot{\theta})=Z / V$. The case shown at the bottom is that treated by Lee (von Hofsten \& Lee, 1982).

eye-this is the major reason tau fails to provide a plausible account of interceptive timing, and $\tau(\dot{\theta})$ fails for the same reason.

Two further problems are associated with $\tau(\dot{\theta})$ : First, it requires the measurement of optical accelerations to which people are known to be insensitive. Stewart et al. (1997) made the curious decision to doubt or consider irrelevant the empirical evidence concerning detection of optical accelerations but did not provide any reasons for doing so; I see no scientific reason for ignoring this finding. Second, in expressions for the immediacy or TTC on bypass approaches such as those discussed above, it is not valid to substitute $\tau(\dot{\theta})$ for $\tau$ (Tresilian, 1997b). However, there is an exception to this that I previously overlooked (though this was not noted by Stewart et al., 1997, in their reply).

Some time ago, Lee proposed that time to nearest approach (TTN, defined at the bottom of Figure 4) might be used for bypass approaches and could be obtained by exploiting the following relation (von Hofsten \& Lee, 1982):

$$
\mathrm{TTN}=\tau /(2+\dot{\tau})
$$

Since $\dot{\tau}=1-\tau \dot{\theta} / \ddot{\theta}$, we can use the notation $\dot{\theta} / \ddot{\theta}=\tau(\dot{\theta})$ and $\Gamma_{N}=1 /$ TTN to rewrite Equation 5 as follows:

$$
\Gamma_{N}=3 \tau^{-1}-\tau(\dot{\theta})^{-1} \text {. }
$$

Lee's suggestion can, therefore, be interpreted as the proposal that $\tau$ and $\tau(\dot{\theta})$ are combined to obtain timing infor- mation for use in bypass approaches. Note that Equations 5 and 6 involve image acceleration, which means that the timing information described by these equations does not neglect acceleration of the target. The reason for this is straightforward: From Figure 4 it follows that $R^{2}=X^{2}+$ $D^{2}$, which yields $R \dot{R}=X \dot{X}$ and $R \ddot{R}+\dot{R}^{2}=X \ddot{X}+\dot{X}^{2}$ when differentiated once and twice, respectively. Equation 5 then follows directly from division of the first derivative by the second if we assume that $\ddot{X}=0$ and use the notation $\tau=$ $-R / \dot{R}, \mathrm{TTN}=-X / \dot{X}$. If $\ddot{X} \neq 0$, then Equation 5 becomes

$$
1-X \ddot{X} / \dot{X}^{2}=\tau(2+\dot{\tau}) \text {. }
$$

Because of this, $\tau /(2+\dot{\tau})$ gives a better approximation to the actual TTC than $-X / X$ when the target object is accelerating, just as $\tau(\dot{\theta})$ gives a better approximation than $\tau$ when the target is accelerating. Thus, $\tau(\dot{\theta})$ could be used for timing interceptions of objects on bypass approaches. Unfortunately, people do not seem to use this information.

In an experiment reported in Tresilian (1994b), participants had their heads held in a fixed position and were required to make a fictive interception ${ }^{2}$ of a self-luminous ball on a bypass approach as it rolled down a trackway in a completely blacked out room. Interceptions were made at the point of nearest approach to the eyes, a point $40 \mathrm{~cm}$ in front of and $40 \mathrm{~cm}$ behind the point of nearest approach. If the time-to-nearest approach were the information used to time interceptions of objects on bypass approaches, as 
Lee had suggested (von Hofsten \& Lee, 1982; Lee \& Young, 1985), then the timing error should have been larger at the points $40 \mathrm{~cm}$ from the nearest approach position. It was found that the errors were small (mean constant error across participants $\approx 10 \mathrm{msec}$ ) and did not differ in the three positions. Tresilian (1994a) concluded that TTN information was not (generally) used to time interceptions of objects on bypass approaches. The experiments reported in Tresilian (1994a) establish that participants were not timing interceptions on the basis of $\tau, \tau(\dot{\theta})$, or TTN.

\section{Combined Use of Monocular and Binocular Cues to TTC}

There is now a body of data that supports the notion that binocular and monocular cues are combined in the estimation of TTC by the human visual system (Gray \& Regan, 1996; Heuer, 1993; Wann \& Rushton, 1995). It is still unclear, however, exactly what sources of information are actually combined in the control of interceptions. A particularly interesting result has recently been obtained in an unpublished experiment conducted by Mark MonWilliams and John Wann. Participants in this experiment were required to hit balls by moving their right hands across the path of the ball, which swung toward them on a length of string (the ball was the "bob" of a pendulum). Balls were released from three different heights, and participants were positioned so that the ball was on a collision course with a point midway between their eyes (needless to say, a mechanical stop prevented such a collision from actually occurring). The hand was initially unseen but became visible as it crossed the path of the swinging ball. (The hand moved approximately perpendicular to the plane of ball motion in a frontoparallel plane a fixed distance in front of the participant). The timing error was calculated as the time difference between the moment a small infrared emitting diode (IRED) attached to participants' right hands crossed the ball's path and the moment an IRED attached to the ball crossed the plane of hand motion (IRED position was monitored using a Selspot ${ }^{\mathrm{TM}}$ movement recording system at $200 \mathrm{~Hz}$ ).

Participants performed the task under four different conditions: (1) normal vision (baseline), (2) looking through cyclopean spectacles (Figure 5, upper right panel), (3) through telestereoscopes (Figure 5, lower right panel), and (4) and through path-lengthening spectacles. Telestereoscopes were devised by Helmholtz and have the effect of increasing the interpupilliary distance and hence the magnitude of binocular disparities. Cyclopean spectacles effectively reduce the interpupiliary distance to zero and thus remove all binocular disparity. Both types of spectacles increase the distance that light must travel from object to eye; Condition 4 was included as a control for this path-lengthening effect of cyclopean spectacles and telestereoscopes (Figure 5, right). The average results,

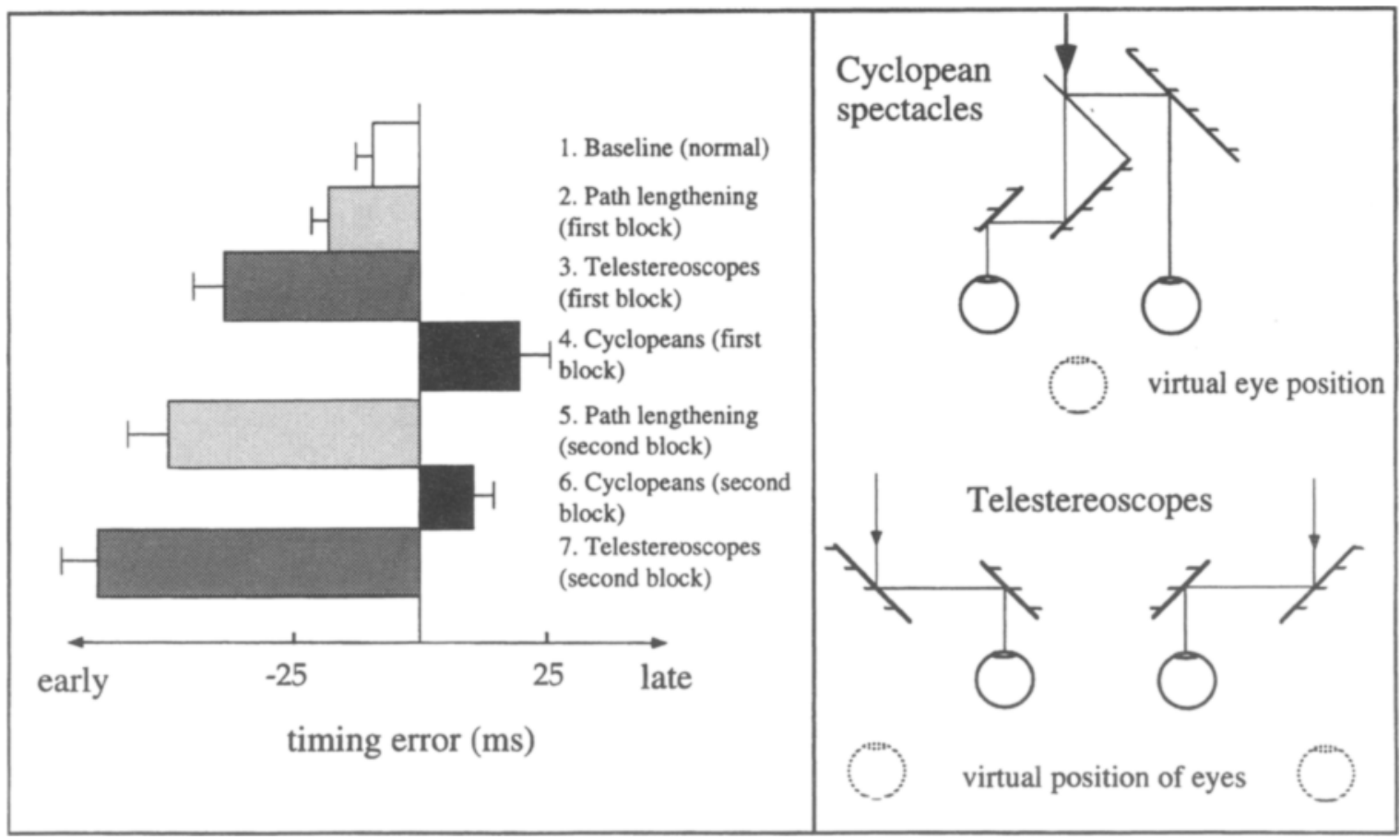

Figure 5. Left: results of experiment conducted by Wann and Mon-Williams (data kindly provided by Mark MonWilliams). Eight participants performed seven blocks of trials under four conditions in the order shown in the legend (left). The group mean timing error for each block is shown (error bars indicate $+1 S D$ ). These means include the effects of adaptation to the optical devices since they are means of blocks of trials. On the right are shown ray diagrams for cyclopean spectacles (top) and telestereoscopes (bottom). In the experiment, the latter were arranged in such a way that the effective interpupilliary distance was approximately twice the normal distance. 
provided by Mark Mon-Williams, are plotted in the left panel of Figure 5. Participants performed the hitting task under the various conditions in the order shown in the figure legend (which provides further details). The important features of the data presented in Figure 5 are the timing errors observed when participants looked through the cyclopeans and telestereoscopes: Through telestereoscopes, participants moved too early, and through cyclopeans, they moved too late. Use of TTC information cannot completely account for these results.

The effect of looking through cyclopean spectacles can be predicted from use of a combination of binocular and monocular TTC information: Binocular information here specifies that TTC is large (greater than can be detectedthe immediacy is subthreshold), whereas monocular information specifies TTC veridically, and any combination of the two (even with much greater weight attached to monocular sources) is expected to result in people responding too late. The effect of looking through telestereoscopes is not expected since these have no effect on either monocular or binocular TTC information so far described in the literature.

One possible way to interpret the telestereoscope result follows from a discussion of TTC information during direct approach to the eye(s), presented in an earlier article (Tresilian, 1991). There I discussed the case of how accurate timing is possible for an interception made some distance in front of the head when the target is moving directly toward the eyes. Pilot observations made prior to the series of experiments reported in Tresilian (1994b) convinced me that $I$ and all others tested were immediately capable of very accurate timing (better than $10 \mathrm{msec}$ ) when a target moving directly toward the eyes was intercepted at a fixed position about $40 \mathrm{~cm}$ in front of the head. All that was visible was the target-a self-luminous ball moving in a completely blacked-out environment. A trapping task not dissimilar to the task used by Mon-Williams and Wann was used for these pilot observations (for details see, Tresilian, 1994b, Experiment 1). The ability to time accurately in these conditions suggests that TTC with the interception point is used; to obtain it, information about the position of the hand is required, along with information about the speed of the target. In these circumstances, the TTC with the interception point is given by (TTC with the eye) - (distance of hand from eye) $\div$ (speed of target) or $T_{i}=T_{e}-d / V$.

Binocular information about the speed of motion in depth is available to an observer: The distance of a binocular target is equal to $(I / 2) \cot \gamma / 2$, where $I$ is the interocular separation and $\gamma$ is the binocular parallax. The target's speed, $V$, is thus the derivative of this $(=I \dot{\gamma} /[4(1-\cos$ $\gamma)]$ ) and so involves $I$, which is increased by the telestereoscopes. The observer must use knowledge of $I$ to determine speed; the value used will be insufficiently large (being close to the value for the eyes, not the value induced by wearing the telestereoscopes), and the target will thus appear to be moving more slowly than it actually is. This will lead to an underestimation of TTC and a re- sponse that is too early. To put this another way, the telestereoscopes change the $d / V$ contribution to the TTC estimate. If $I_{T}$ is the interocular separation and $\gamma_{T}$ is the binocular parallax when one is wearing telestereoscopes, then $d / V=4 d\left(1-\cos \gamma_{T}\right) /\left(\dot{\gamma}_{T} I_{T}\right)$; the nervous system can measure $\gamma_{T}$ and $\dot{\gamma}_{T}$ but "assumes" $I_{T}=I$ prior to adaptation. Note that $d$ must be estimated using limb proprioception since the hand is initially out of the field of view. Since $I<I_{T}$ the estimate of $d / V$ will be too large and hence $T_{e}$ less this estimate, too small. In the experiment of MonWilliams and Wann, $I_{T}$ was twice $I$ : The effect of this is to double the estimate of $d / V$. The expected error in timing the hitting response can be roughly estimated as follows: The speed of the ball was fairly constant at about 4 to $5 \mathrm{~m} / \mathrm{sec}$ over the final $600 \mathrm{msec}$ of its approach to the interception point. For $d=0.4 \mathrm{~m}$, the actual value of $d / V$ is then between about 80 and $100 \mathrm{msec}$ : For $I_{T}=2 I$, the telestereoscope estimate is double the normal estimate and so the expected error will be about $90 \pm 10 \mathrm{msec}$. Clearly it will vary with $d$ : For $d=0.3 \mathrm{~m}$, the expected error is about $65 \pm 10 \mathrm{msec}$. In the experiment of Mon-Williams and Wann, $d$ was approximately $0.3 \mathrm{~m}$. The expected timing error would then be in the region of $65 \mathrm{msec}$. This is much larger than the error shown on the bar graph in Figure 5, which is about $35 \mathrm{msec}$ (telestereoscopes first block), which reduces to about $17 \mathrm{msec}$ when the path-lengthening effect of the telestereoscopes is taken into account: (telestereoscopes first block) - (path-lengthening first block). The error shown in the figure, however, is the mean of 27 consecutive trials and therefore includes the effects of adaptation to the spectacles (the error decreased over repeated trials as participants adapted). The error due to the telestereoscopes (corrected for path lengthening) in the first two trials of the block was, averaged across participants, about $60 \mathrm{msec}$. This level of quantitative agreement makes it plausible to suggest that the telestereoscopes affected the timing of participants' responses in the experiment described because the $d / V$ correction to TTC with the head is computed using binocular information from the target object and proprioceptive information about limb position.

\section{ARE TIMING AND POSITIONING REALLY SEPARABLE?}

To hit a moving ball, for example, the hand must arrive at a position on the ball's path at the same time as the ball. Position and time are obviously mutually dependent: Each position on a ball's path is associated with a particular time of arrival of the ball - the path is time parameterized; this what it means to be a trajectory. A particular time-toarrival determines a particular position and vice versa. In this sense, therefore, time and position are inseparable. The sense of separability at issue here, however, has to do with whether different types of information control the timing and positioning of a movement. The use of independently derived timing and positioning information does not imply that during task execution, timing and positioning 
are controlled by separate mechanisms (though this may be the case).

That explicit timing information is not necessary to successfully intercept moving targets is evidenced by the fact that simple feedback control schemes can reliably achieve interception. To take a nautical example, one ship can intercept the path of a second ship if the pilot of the first ship is moving sufficiently fast and keeps the second ship on a constant bearing. In fact, von Hofsten has put forward a scheme of this type to account for the ability for seated individuals to reach out and grab objects moving along a linear path (von Hofsten \& Lee, 1982). However, this scheme cannot, in principle, account for catching performance under these conditions because of the constraint that the hand should not move further than reachable distance (see Tresilian, 1990, 1993). Constant bearing feedback schemes will work generally only if there are no constraints on the distance the intercepting effector can move. A situation in which this desideratum is met satisfactorily is found when people need to move to intercept a projectile, as in the case of an outfielder in baseball running to catch a flyball. In this case, it has been proposed that a feedback control strategy could be used, obviating the need for detection of time and distance (Chapman, 1968; McBeath et al., 1995; McLeod \& Dienes, 1993; Micheals \& Oudejans, 1992).

\section{Are Timing and Positioning Inseparable in Running to Intercept an Object?}

Chapman (1968) showed mathematically that a fielder who wants to move to intercept a ball projected under gravitational acceleration (no air resistance) can do so if he/she moves at a constant speed that keeps the rate of change of the tangent of the ball's elevation angle constant. This is equivalent to keeping the acceleration of the tangent, $d^{2}(\tan \alpha) d t^{2}$, equal to zero. Thus, the fielder need only measure $d^{2}(\tan \alpha) d t^{2}$ and implement a proportional feedback control law, which tends to cause a change in his/her velocity, which makes $d^{2}(\tan \alpha) d t^{2}$ tend to zero. Thus, no detection of time-to-arrival or prediction of the ball's path is necessary. More recent work has demonstrated that Chapman's scheme can account for the performance of a human fielder in real-world conditions (Dienes \& McLeod, 1993; Tresilian, 1995b).

Despite the collection of a considerable quantity of empirical data and debate about the precise nature of the feedback control scheme used (McBeath et al., 1995; McLeod \& Dienes, 1993, 1996; Micheals \& Oudejans, 1992), one very important point has been virtually overlooked. There are two proposals about how fielders intercept projectiles: the Chapman-McBeath type of strategy and strategies based on predictive information about where the projectile is going and when it is going to get there. Both of these strategies result in $d^{2}(\tan \alpha) d t^{2}$ being approximately zero during a fielder's run to catch a ball (Tresilian, 1995b), so the experimental demonstration that $d^{2}(\tan \alpha) d t^{2}$ is approximately zero cannot distinguish between the alternatives. Indeed, keeping $d^{2}(\tan \alpha) d t^{2}$ close to zero guarantees that a fielder will be in the right place at the right time to intercept a ball: If a fielder runs in such a manner that the ball is successfully caught, $d^{2}(\tan \alpha) d t^{2}$ will be close to zero. In other words, demonstrating that $d^{2}(\tan \alpha) d t^{2}$ is approximately zero tells us little more than that the ball was successfully intercepted. Since the empirical data used in support of Chapman's (1968) strategy is restricted to demonstration of $d^{2}(\tan \alpha) d t^{2}$ being zero (McLeod \& Dienes, 1993, 1996; Micheals \& Oudejans, 1992), we can conclude little, if anything, about how a fielder actually guides movement when running to catch a ball.

McLeod and Dienes (1996) made the claim that if the fielder knew where to go and was then given plenty of time, "running more slowly and arriving just in time to catch the ball would seem a pointlessly risky strategy. Why not go to the right place and wait?" (p. 535). What people actually tend to do is arrive just in time rather than go somewhere and wait. McLeod and Dienes (1996) took this to be conclusive evidence that fielders do not use information about where the projectile is going to control their running. However, it has not actually been established that arriving just in time is pointlessly risky if fielders use information about where the projectile is going. It certainly seems pointlessly risky, as McLeod and Dienes (1996) observed, but why? The reason is probably due to the use of a bus stop type analogy. We want to catch the bus: We know where the bus will be (at the bus stop) and a timetable tells us when it will get there. It would be pointlessly risky to arrange our trip to the bus stop so that we arrive there just as the bus is arriving - it would be much more sensible to go there and wait. Use of this as an analogy for projectile interception assumes that we know exactly where the projectile will be and when it will get there. But this is not an assumption shared by a predictive interception strategy. It is completely implausible that fielders would have accurate information about where a ball is going and when it will get there. Theoretical work and empirical data suggest that the information would be relatively inaccurate early in the ball's flight and improve progressively as it approached its landing position (see, e.g., Saxberg, 1987a, 1987b; Todd, 1981). ${ }^{3}$ It would be foolish for a fielder to wait until he/she has a good idea of where the ball is going before starting to move, because by that stage there would often not be enough time to get to the ball before it landed. This violates McLeod and Dienes's (1996) assumption that the fielder has plenty of time. It would be better to start running with only a rough idea of where the ball is going and then repeatedly refine the estimate en route. Use of such a strategy is quite consistent with the observation that people do not go to the right place and wait for the ball's arrival.

I recently reported the results of an attempt to distinguish empirically between the two strategies for projectile interception (Tresilian, 1995b). Existing theoretical analyses have demonstrated that information about where the projectile is going in the absence of air resistance effects is available to an observer (Saxberg, 1987a; Todd, 1981; Tresilian, 1993). A knowing-where-to-go strategy based on this information would lead to fielders who were not initially positioned in the plane of projectile motion, mov- 
ing to an interception along a concave path relative to the plane of projection (a consequence of ignoring air resistance effects). Simulations of Chapman's (1968) strategy and variants of it designed to work when the fielder is not initially in the plane of projection (see Tresilian, 1995b) suggested that concave paths would not be observed for fielders implementing this strategy - the paths should instead tend to be convex. People were filmed running to catch balls that were thrown to the left or right of their initial position (participants were not initially in the plane of projection but were displaced by up to $5 \mathrm{~m}$ from the plane to either the left or the right). Two balls were used: a tennis ball and a sponge ball of similar size and color but with a smaller mass and a greater drag coefficient. A predictive strategy based on information that ignores air resistance should produce paths of greater concavity for the sponge ball. Paths were approximately straight, irrespective of the ball used, and similar results were reported by McBeath et al. (1995). These results offer no support for the use of information about where the ball is going that ignores air resistance.

In summary, it can be tentatively concluded that fielders do not rely on information that ignores air resistance. We cannot conclude from this that they use Chapman's (1968) strategy or something similar (cf. McBeath et al., 1995), as this would be to commit the logical error of affirming the consequent. Indeed, we have no evidence that Chapman's strategy is used (see above); we simply lack evidence that it is not used. I believe that the only studies that directly attempted to obtain evidence against Chapman's strategy were reported in Tresilian (1995b), one empirical and one theoretical. The empirical study allowed only the conclusion that fielders did not use a knowingwhere strategy based on information that ignored air resistance. The theoretical study attempted to determine whether visual insensitivity to accelerations would preclude effective implementation of Chapman's strategy by a human fielder; the answer was no. I do not believe that these results can be used to support the contention that people use Chapman's strategy. Given the lack of empirical evidence, an appeal to the principle of parsimony would dictate that we accept the knowing-where strategy in preference to Chapman's strategy.

\section{SUMMARY AND CONCLUSIONS}

I have recently attempted to provide an approach to understanding how interceptive actions are timed that is consistent with both the remarkable timing skills displayed by people in fast ball games and the corpus of experimental data relevant to these and other timing skills (Tresilian, 1994b, 1995a). Four challenges to some of the basic assumptions of my approach have appeared and these were considered here. (1) The approach assumes that there are cues to TTC available in the stimulus and formal analysis of stimulus geometry has demonstrated the existence of these cues. However, this analysis was confined to a sta- tionary observer, which raises some potentially important problems. It was shown that it is possible to generalize the analysis to the case of a moving observer and so avoid the difficulties encountered by previous analyses. (2) Smeets and colleagues (1996) recently presented data interpreted as demonstrating that people compute TTC from independently derived information about distances and velocities in 3-D space. It was shown that Smeets and colleagues data are actually more parsimoniously accounted for by the hypothesis that people use TTC information. Also discussed was a recent experiment conducted by Mon-Williams and Wann that demonstrated that transiently increasing the interocular separation (by wearing telestereoscopes) led to early responses in a hitting task. (3) Stewart and colleagues $(1993,1997)$ have recently questioned the assumption that image accelerations are not used in the computation of TTC for fast interceptions. They have presented an alternative source of TTC information - the ratio of image speed to image acceleration. Theoretical problems with Stewart et al.'s analysis have been previously identified (Tresilian, 1997b). It was shown that even if these could be overcome, the alternative information proposed by Stewart et al. cannot account for the available experimental data. (4) Some models of projectile interception raise questions concerning the necessity of treating timing and positioning as separable components of interception. These models work for fielders moving to get into the right place to catch a projectile. The problem is that extant data do not allow us to distinguish whether people use one of these feedback strategies or a predictive strategy involving perception of where the projectile is going and when it will get there-currently we simply lack evidence that people do not use a feedback strategy. In these circumstances, the principle of parsimony argues that the predictive strategy is the more acceptable hypothesis.

It can be concluded that an existing hypothesis of how interceptions are timed can accommodate extant data and provides a more parsimonious account than the alternatives. This hypothesis represents a radical development of Lee's tau hypothesis. In many respects, it departs substantially from Lee's hypothesis: Even where features of Lee's hypothesis are retained (e.g., tau variables are used in timing, and accelerations are ignored in timing fast interceptions), the rationale for these features differs, and they are subject to qualification. Although the new hypothesis may be wrong, its detailed empirical and logical evaluation will further understanding of the information and processes involved in timing interceptions. Certainly, it is the only hypothesis currently specified in the literature that is capable of accounting for existing data in a systematic and unified fashion.

\section{REFERENCES}

BABler, T. G., \& DANNemillar, J. L. (1993). Role of image acceleration in judging landing location of free-falling projectiles. Journal of Experimental Psychology: Human Perception \& Performance, 19, 15-31.

Bootsma, R. J., \& OUdEJANS, R. (1993). Visual information about time 
to collision between two objects. Journal of Experimental Psychology: Human Perception \& Performance, 19, 1041-1052.

Bootsma, R. J., \& VAN Wieringen, P. C. W. (1990). Timing an attacking forehand drive in table tennis. Journal of Experimental Psychology: Human Perception \& Performance, 16, 21-29.

Brenner, E., \& SMEETS, J. B. J. (1994). Why we hit slow targets more gently. Journal of Physiology, 479, 53P.

Calderone, J. B., \& KaISER, M. K. (1989). Visual acceleration detection: Effect of sign and motion orientation. Perception \& Psychophysics, 45, 391-394.

Chapman, S. (1968). Catching a baseball. American Journal of Physics, $36,868-870$.

Davis, M. N. O., \& GREEN, P. (1990). Optic flow-field variables trigger landing in hawk but not in pigeons. Naturwissenschaften, 77, 142-144.

DELUCIA, P. R. (1995). Effects of pictorial relative size and ground intercept information on judgments about potential collisions in perspective displays. Human Factors, 37, 528-538.

DeLucia, P. R. \& MEYer, L. E. (1997). Time to contact judgements in the presence of observer motion [Abstract]. Investigative Ophthalmology \& Visual Science, 38, S79.

DeLuCIA, P. R. \& MEYER, L. E. (in press). Judgments about the timeto-contact between two objects during simulated self-motion. Journal of Experimental Psychology: Human Perception \& Performance.

Dienes, Z., \& MCLEoD, P. (1993). How to catch a cricket ball. Perception, 22, 1427-1439.

Gray, R., \& REGAN, D. (1996). Estimates of time to collision based on binocular and monocular visual information. Investigative Ophthalmology \& Visual Science, 37 (Suppl.), S652.

HeUER, H. (1993). Estimates of time to contact based on changing size and changing target vergence. Perception, 22, 549-563.

Hofsten, C. vON, \& LEE, D. N. (1982). Dialogue on perception and action. Human Movement Science, 1, 125-138.

KatSER, M. K., \& MoWAFY, L. (1993). Optical specification of time-topassage: Observer's sensitivity to global tau. Journal of Experimental Psychology: Human Perception \& Performance, 19, 1028-1040.

KOENDERINK, J. (1985). Space, form and optical deformations. In D. Ingle, M. Jeannerod, \& D. N. Lee (Eds.), Brain mechanisms and spatial vision (pp. 31-55). Dordrecht: Martinus Nijhoff.

LaCQUANITI, F., \& MAFOLI, C. (1989). The role of preparation in tuning anticipatory and reflex responses during catching. Journal of Neuroscience, 9, 134-148.

Laurent, M., Montagne, G., \& Savelsbergh, G. J. P. (1995). The control and coordination of one-handed catching: The effect of temporal constraints. Experimental Brain Research, 101, 314-322.

LEE, D. N. (1976). A theory of visual control of braking based on information about time-to-collision. Perception, 5, 437-459.

LEE, D. N. (1980). Visuo-motor coordination in space-time. In G. E. Stelmach \& J. Requin (Eds.), Tutorials in motor behavior (pp. 281292). Amsterdam: North-Holland.

LEE, D. N., \& REDDISH, P. E. (1981). Plummeting gannets: A paradigm of ecological optics. Nature, 293, 293-294.

LEE, D. N., \& YOUNG, D. S. (1985). Visual timing of interceptive action. In D. Ingle, M. Jeannerod, \& D. N. Lee (Eds.), Brain mechanisms and spatial vision (pp. 1-30). Dordrecht: Martinus Nijhoff.

LeE, D. N., Young, D. S., Reddish, P. E., Lough, S., \& Clayton, T. (1983). Visual timing in hitting an accelerating ball. Quarterly Journal of Experimental Psychology, 35A, 333-346.

MCBeath, M. K., Shaffer, D. M., \& Kaiser, M. K. (1995). How baseball outfielders determine where to run to catch fly balls. Science, 268, 569-573.

MCLEOD, P. (1987). Visual reaction time and high speed ball games. Perception, 16, 49-59.

MCLEOD, P., \& DIENES, Z. (1993). Running to catch a ball. Nature, 362, 23.

MCLEOD, P., \& DiENES, Z. (1996). Do fielders know where to go to catch the ball or only how to get there? Journal of Experimental Psychology: Human Perception \& Performance, 22, 531-543.

Micheals, C. F., \& Oudejans, R. (1992). The optics and actions of catching fly balls: Zeroing out optical acceleration. Ecological Psychology, 4, 199-222.
RAMACHANDRAN, V. S. (1990). Visual perception in people and machines. In A. Blake \& T. Troscianko (Eds.), Al and the eye (pp. 21-35). Chichester, U.K.: Wiley.

REGAN, D. (1992). Visual judgements and misjudgements in cricket, and the art of flight. Perception, 21,91-115.

REgan, D., \& HaMSTRA, S. J. (1993). Dissociation of discrimination thresholds for time to contact and for rate of angular expansion. Vision Research, 33, 447-462.

Regan, D., Hamstra, S. J., Kaushal, S., Vincent, A., Gray, R., \& BEVERLEX, K. I. (1995). Visual processing of the motion of an object in three dimensions for a stationary or a moving observer. Perception, 24, 87-103

Regan, D., Kaufman, L., \& Lincoln, J. (1986). Motion in depth and visual acceleration. In K. R. Boff, L. Kaufman, \& J. P. Thomas (Eds.), Handbook of perception and human performance: Vol. I. Sensory processes and perception (pp. 19-1 to 19-46). New York: Wiley.

SAXBERG, B.V. H. (1987a). Projected free-fall trajectories: I. Theory and simulation. Biological Cybernetics, 56, 159-175.

SaXberG, B.V. H. (1987b). Projected free-fall trajectories: II. Human experiments. Biological Cybernetics, 56, 177-184.

SCHIFF, W., \& DETWILER, M. L. (1979). Information used in judging impending collision. Perception, 8, 647-658.

Smeets, J. B. J., Brenner, E., Trebuchet, S., \& Mestre, D. R. (1996). Is judging time-to-collision based on 'tau?' Perception, 25, 583-590.

Stewart, D., Cudworth, C., \& Lishman, J. R. (1993). Misperception of time-to-collision by drivers in pedestrian accidents. Perception, 22 , 1227-1244.

Stewart, D., Cudworth, C., \& Lishman, J. R. (1997). Correcting some misperceptions of time-to-collision response. Perception, 26, 237-241

ToDD, J. T. (1981). Visual information about moving objects. Joumal of Experimental Psychology: Human Perception \& Performance, 7, 795-810.

TREsilian, J. R. (1990). Perceptual information for the timing of interceptive action. Perception, 19, 223-239.

Tresilian, J. R. (1991). Empirical and theoretical issues in the perception of time to contact. Journal of Experimental Psychology: Human Perception \& Performance, 17, 865-876.

TREsilian, J. R. (1993). Four questions of time to contact: A critical examination of research on interceptive timing. Perception, 22, 653-680.

Tresilian, J. R. (1994a). Approximate information sources and perceptual variables in interceptive timing. Journal of Experimental Psychology: Human Perception \& Performance, 20, 154-173.

Tresilian, J. R. (1994b). Perceptual and motor processes in interceptive timing. Human Movement Science, 13, 335-373.

Tresilian, J. R. (1995a). Perceptual and cognitive processes in time-tocontact estimation: Analysis of prediction-motion and relative judgment tasks. Perception \& Psychophysics, 57, 231-245.

Tresilian, J. R. (1995b). Study of a servo control strategy for projectile interception. Quarterly Journal of Experimental Psychology, 48A, 688-715.

Tresilian, J. R. (1997a). Correcting some misperceptions of time-tocollision: A critical note. Perception, 26, 229-236.

TRESILIAN, J. R. (1997b). The revised tau hypothesis: A consideration of Wann's analyses. Journal of Experimental Psychology: Human Perception \& Performance, 23, 1272-1281.

VAN DeR WeEL, R., VAN DER MeER, A., \& LeE, D. N. (1996). Measuring basic perceptuo-motor dysfunction in cerebral palsy. Human Movement Science, 15, 671-689.

Wallace, S., Stephenson, E., Weeks, D., \& Kelso, J. A. S. (1992). The perceptual guidance of grasping a moving object. Human Movement Science, 11, 691-716.

WANN, J. P. (1996). Anticipating arrival: Is the tau-margin a specious theory? Joumal of Experimental Psychology: Human Perception \& Performance, 22, 1031-1048.

WANN, J. P., \& RusHToN, S. (1995). Grasping the impossible: Stereoscopically presented virtual balls. In B. Bardy, R. J. Bootsma, \& Y. Guiard (Eds.), Studies in perception and action III (pp. 207-210). Hillsdale, NJ: Erlbaum

WerkHoven, P., SNIPPE, H. P., \& TOET, A. (1992). Visual processing of optic acceleration. Vision Research, 32, 2313-2329. 


\section{NOTES}

1. Note that this is different from the argument of Lee (Lee \& Reddish, 1981; Lee et al., 1983). Lee proposed that object accelerations are ignored because this is a corollary of the "simple" strategy of using tau as timing information. Indeed, Lee has proposed that image accelerations may be used in the computation of TTC (von Hofsten \& Lee, 1982). My argument has nothing to do with "simplicity"--the conception of TTC perception I have proposed is actually rather complex (Tresilian, 1994a). The argument is based on psychophysical data that strongly suggest that in the time available, estimates of image acceleration could not contribute to accurate timing of fast interceptive actions (Babler $\&$ Dannemillar, 1993; Calderone \& Kaiser, 1989; Regan, Kaufman, \& Lincoln, 1986; Werkhoven, Snippe \& Toet, 1992).

2. Participants were instructed to bring their right hands down onto a contact switch positioned on a perspex sheet about $3 \mathrm{~cm}$ directly above the path of the moving target (ball).

3. McLeod and Dienes (1996, p. 531) made an argument concerning the early flight information available to a person. They considered paths of balls projected toward a fielder at the same angle from the same position with speeds so that they land either at $5 \mathrm{~m}$ behind or $5 \mathrm{~m}$ in front of the fielder. These paths are very similar for the first $800 \mathrm{msec}$ or so of the ball's flight. From this observation, it was suggested that there is "insufficient information to work out where the ball will land," which is "paradoxical if the fielder is assumed to know where the ball will land when starting to run" (p. 534). However, the argument presented does not establish this, since it was shown only that the paths of the balls were very similar-their speeds over the first $800 \mathrm{msec}$ will be different. The initial visual angular velocities of the three projectiles in McLeod and Dienes's example are $20 \% \mathrm{sec}, 21.6 \% \mathrm{sec}$, and $23.1 \% \mathrm{sec}$. In any event, the trajectories were evidently sufficiently different for the fielder to start running in the right direction; this could easily be because he/she has a sufficiently good idea of where the ball is going to be able to do this.

(Manuscript received August 6, 1997; revision accepted for publication February 26, 1998.) 